\title{
Abstracts of the 23rd Annual Brain Impairment Conference 12-14 May 2000, Melbourne
}

\section{WORKSHOP 1}

\section{Effects of Unilteral Versus Bilateral Pathology on the Development of Speech and Language Functions in Children}

Faraneh Vargha-Khadem

Cognitive Neuroscience Unit, Institute of Child Health, University College

London Medical School, and Great Ormond Street Hospital for Children, London, UK

$\mathrm{U}$ nlike adults, who show chronic and severe aphasic symptoms after focal left cortical and/or subcortical damage, children rarely demonstrate persistent disruption of speech and language functions following such lesions. Laterality of the lesion does not appear to be a critical factor in determining gross speech and language outcome, provided the injury is sustained before the age of puberty. Therefore, in both developmental and acquired disorders where speech and language functions are selectively and permanently compromised, bilateral pathology of one or more components of the perisylvian areas or of the subcortical structures with which they are directly connected must be suspected.

Data obtained from large groups of children with acute stroke or ischaemic damage, and those with hemispherectomy will be presented to demonstrate how different aspects of speech and language functions are preserved, in line with expectations derived from overall intellectual ability, regardless of hemispheric side of damage or removal, or age at injury (i.e. congenital or acquired during childhood). These data will be used to illustrate the remarkable capacity of the immature brain for functional reorganisation and for rescuing of cognitive abilities in the face of early unilateral brain pathology.

As a contrast, a case will be presented to demonstrate that perinatally acquired, bilateral focal pathology of the frontal lobes precludes the development of speech. In addition, data from a large three-generational family with an inherited speech and language disorder will be presented to illustrate that bilateral pathology of the basal ganglia produces orofacial and verbal dyspraxia, the phenotype of this genetic disorder.

\section{WORKSHOP 2}

\section{A Case-Driven Approach to the Neuropsychology of Stroke}

Michael M Saling and Jennifer M Bradshaw

Austin and Repatriation Medical Centre, Austin Campus, Studley Road, Heidelberg, Vic., 3084

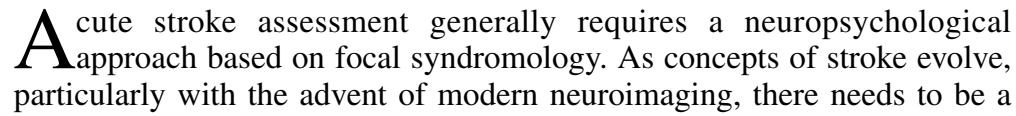


continuing adaptation of the approach to neuropsychological examination in the acute stroke setting.

The aim of this workshop is to provide participants with basic principles on those aspects of stroke relevant to neuropsychology. On completion of the workshop participants will have an appreciation of the aims and principles of bed-side neuropsychological examination and the way in which neuropsychological formulation feeds into the management activities of the stroke team as a whole. The workshop will be of value to all who deal with neuropsychological disorders. Previous experience in stroke is not a prerequisite.

\section{WORKSHOP 3}

\section{Focal Cerebral Degeneration}

Julie Snowden

Cerebral Function Unit, Royal Manchester Infirmary, England

century ago, Arnold Pick described a series of patients in whom a focal neuropsychological syndrome was associated with circumscribed degeneration of the brain. One hundred years on, focal degenerative syndromes are increasingly recognised, although their relationship to each other and to established disease entities remains a source of confusion. Focal syndromes include frontotemporal dementia, progressive aphasia, progressive apraxia, progressive agnosia, posterior cortical atrophy and progressive amnesia. Although in some instances focal neuropsychological syndromes are the precursor to more widespread cognitive impairment, in others the focal disorder persists and is associated with a circumscribed distribution of atrophy demonstrable at autopsy.

The workshop will focus principally on clinical syndromes which are associated with non-Alzheimer's disease pathology and result from circumscribed degeneration of the frontal and/or temporal lobes: frontotemporal dementia, progressive aphasia and semantic dementia. Historical, neurological, neuropsychological and neuroimaging characteristics of these clinical disorders will be described and video case presentations will be used to illustrate salient behavioural and cognitive features. A discussion of the underlying pathology will focus particularly on the inter-relationship between the distinct syndromes and their relationship to "Picks disease". Clinical comparisons will be made with Alzheimer's disease. Focal cerebral degeneration can give rise to remarkably circumscribed neuropsychological deficits, which have the potential to shed light on normal brain functions. This will be illustrated by a discussion of the breakdown of semantic memory, and its implications for the relationship between memory systems.

The workshop aims to raise awareness of distinct clinical syndromes associated with focal degeneration of the frontotemporal lobes and to provide a framework for their clinical recognition and differentiation from Alzheimer's disease. Contemporary nosological issues in the field of dementia will be addressed. 


\title{
WORKSHOP 4
}

\section{“Now I'm feeling sorry for the pixies." The Diagnosis and Management of Attention Deficit Disorder}

David Stokes, Vicki Anderson and Lionel Lubitz

Department of Psychology, Royal Melbourne Institute of Technology

\begin{abstract}
The workshop will focus on the diagnosis and management of children 1 with ADHD. The presentations will involve two clinical neuropsychologists and a consultant paediatrician. The clinical neuropsychologists will cover the complex issues of attention, hyperactivity and executive function, discuss their assessment and the tests that best operationalise their meaning. Case examples will be used to expand on this process and to raise a selection of difficulties commonly found in the process of diagnosis. Management will be mainly looked at from a medical viewpoint including current medication, supporting parents and the interface of neuropsychology and paediatrics. There will be opportunity for questions and discussion.
\end{abstract}

SYMPOSIUM 1: GREY MATTER, LIFE

MATTERS, HEART MATTERS: WHAT MATTERS?

James Bennett-Levy

Southern Cross University, Standing Street, The Channon, NSW 2480

Chris Williams

North Coast Head Injury Service, 56 Uralba Street, Lismore, NSW 2480

\begin{abstract}
cquired brain injury frequently results in deleterious outcomes. AEveryday life is affected in various ways: relationships, work, home life, independence and self-determination. For some, even the opportunity to lead an everyday life is under threat. Our clients may lose heart. As professionals, our perspective and behaviour significantly impact on the communications we make with people, with our clients, and the kinds of service we deliver. Consumer satisfaction and rehabilitation outcomes may be affected. The medical model, still the dominant rehabilitation discourse, may not be well-suited to the delivery of effective emotional, cognitive and psychosocial rehabilitation in everyday life. Alternative models, dependent on different values and assumptions, may be more empowering both for clients and professionals. In this symposium, we shall seek to (i) elucidate some of the often unquestioned perspectives inherent in professionals' behaviour and (ii) give a number of practical examples of alternative ways to facilitate the rehabilitation process for people who have sustained damage to the neurological system.
\end{abstract}

\section{Values and Goals, Glasses and Roles: The Impact of Professional's Thinking and Behaviour on People With Neurological Disabilities}

James Bennett-Levy

Southern Cross University, Standing Street, The Channon, NSW, 2480

Tn the past 20 years, the perspective and practices of rehabilitation professionals have changed considerably. The author undertakes a light-hearted review of his professional life as a researcher, neuropsychologist and clini- 
cal psychologist in the field of neurological rehabilitation. He concludes that the effects of professional training, and the systems in which we work, may act to limit the benefits of rehabilitation for people with neurological disabilities. Sometimes it is necessary to question the values underpinning our professional training; unlearn associated behaviours; and develop more adaptive ones, in order to deliver a more effective service.

\title{
The Things That Matter: An Alternate Model for Value Added Rehabilitation in the Community
}

Chris Williams and Vicki Solomon

North Coast Head Injury Service, Port Macquarie, Shop 7,

Port Pacific Building, Clarence Street, Port Macquarie, NSW 2444

Working effectively with people who have severe brain injury and their families and friends in regional Australia, with one worker per 13,000 sq kms, represents a significant challenge. One aspect of this challenge is questioning how our values and assumptions affect the whole rehabilitation process. This paper describes a value-based outreach model designed to meet (i) the everyday needs of service users living in rural communities (ii) the institutional requirements of effective time management, quality assurance and verifiable rehabilitation plans.

\section{Community Living Options Following ABI - A Case Example of a Man with Severe Memory and Learning Difficulties}

Sue Sloan, Anna Belinda Renison and Joan Tierney

103 Eglington Street, Kew, VIC 3101

\begin{abstract}
ccommodation options for people with acquired brain injury are limAited. For those with severe acquired brain injury, options are even fewer, with most individuals living with their families, in nursing homes or supported residential services (Victorian Coalition of ABI Service providers 1998). Given that many people experience their injury in their 20's, these options are often inappropriate and restrictive in enabling people to meet their needs and goals. This paper presents a unique, client-centred accommodation option that was developed for a 23 year old man who had suffered a severe traumatic brain injury resulting in marked cognitive-behavioural impairments. Over an 18 month period, drawing on a student housing model, the client was assisted to move from a locked psychiatric ward to a home shared with non-disabled tenants and a slow-stream rehabilitation program was integrated into his daily routine. To assist in understanding how this option was conceptualised, background information, including neuropsychological and functional data, will be presented. The strengths of the client, his family and the rehabilitation team which had an impact on the development of the accommodation project will be discussed, as well as the barriers encountered. Particular attention will be given to the philosophy of the approach and the supports and strategies put into place in regards to the client's cognitive-behavioural difficulties. It is hoped that this case study will inspire health care professionals to consider a wider range of possibilities when developing accommodation options for young people with acquired brain injury.
\end{abstract}




\title{
Life in a Time Warp. The Right to Live in the Community Despite Severe Adynamia
}

\author{
Donna Carmichael, Jacquelin Boon and Chris Williams \\ North Coast Head Injury Service, 56 Uralba Street, Lismore, NSW 2480
}

\begin{abstract}
A case presentation where the "dignity of risk" concept is used as the starting point to rehabilitation: Would it be fair to allow a 58 year old man with such severe adynamia to trial living alone in the community? What supports would it take? Would it be safe? Would a nursing home or hostel be safer? What if, after the trial period, the level of support needed was no longer available? Is it possible to reduce the effects of adynamia? What if the person failed? Who is responsible?
\end{abstract}

The presentation features a model for moving systematically from 24 hour live-in support to, in this case, $5 \mathrm{hrs}$ per day. It discusses the value of community supports. Various technical strategies "gizmos and gadgets" and compensatory strategies are demonstrated. Included is a brief video taken 3 years later, featuring comments by staff of the local inpatient rehabilitation unit, support workers, family and, of course the person himself.

A brief comparison of Neuropsychological Assessment results pre-move to community and 2 years later will also be presented. It will answer "How much is recovery and how much is simply very effective compensation?"

\section{SYMPOSIUM 2: NEW TREATMENT AND ASSESSMENT TOOLS IN MULTIPLE SCLEROSIS}

\section{First-Ever "Treatment" Drugs Now Available to People with MS. This is Good News ...Isn't It?}

Wendy Longley

Multiple Sclerosis Society of NSW, PO Box 210, Lidcombe, NSW 1825

$\mathrm{I}_{\mathrm{s}}^{\mathrm{n}}$ n April 1993 an "expedited publication" by the IFNB Multiple Sclerosis Study Group appeared in Neurology, entitled "Interferon beta-1b is effective in relapsing-remitting multiple sclerosis". Excitement swept the world of MS - a "treatment" at last! In June 1995, the Australian regulatory authorities approved the use of this medicine, known as Betaferon TM within Australia, for ambulatory people with relapsing-remitting MS. More importantly, in June 1996, after intense lobbying from many interested parties, this medicine was listed on the Australian PBS scheme for subsidising. Consequently, Australian citizens, unlike most others around the rest of the world, now pay only $\$ 240 /$ year to obtain this medicine, rather than the "market" cost of \$16,800/year - an amazing opportunity for PwMS within Australia. Three new "treatment" medicines have since also become available in Australia: AvonexTM, CopaxoneTM and Rebif TM - all either already listed on the PBS or likely to be listed soon.

In spite of such positive advances and the initial euphoria, however, these immune modulators have not been universally accepted with glee. This has been a most interesting phenomenon to observe: The scientific evidence for the usefulness of these "partial treatment" medicines has been interpreted, and clinically applied, very differently by health professionals around the world. There are still large discrepancies between countries regarding the 
availability and affordability of these medicines for PwMS. Many people with MS in Australia who are theoretically "eligible" to take these medicines have not chosen to do so, and other people with MS who have been on these medicines without any problems have nevertheless chosen to come off them. Why? In this brief discussion, I will update participants on the fascinating phenomenon of the new "MS treatments", from a (neuro)psychologist's point of view.

\title{
Screening of Cognitive Impairment in MS - Relatively Conflict-Free Use Of New Screening Tool By Occupational Therapists
}

\author{
Megan Dephoff, Terri Johnson, Christa Roessler and Wendy Longley \\ Multiple Sclerosis Society of NSW, PO Box 210, Lidscombe, NSW 1825
}

\begin{abstract}
Cognitive change in Multiple Sclerosis (MS) is caused, mainly, by patches of demyelination that form on nerves in the subcortex of the brain. Relatively common cognitive changes (in up to $40 \%$ of people with MS) include reduced speed of thinking and concentration; poor memory and new learning; diminished problem solving and initiation, and reduced flexibility and insight. Cognitive changes can significantly impact on major life tasks and roles: limitation (or loss) of employment, social interaction, home management and self care activities, and community access. Occupational Therapists help people with MS (PwMS) manage the functional impact of these cognitive changes. However, cognitive change is not always easy to detect within the semi-structured interactions of a rehabilitation setting. Even PwMS who have moderate cognitive change usually also have intact basic attention, language and routine behavioural skills, including good social skills. These PwMS may not have good insight into their cognitive changes, or may not be able to describe their cognitive changes adequately to staff. Hence, one of the first ways that Occupational Therapists can help, is in the area of assessment of cognitive change. Occupational Therapists, working with Neuropsychologists at the MS Society of NSW, routinely assess cognition as part of a comprehensive assessment process.

The Screening Examination For Cognitive Impairment (SEFCI) is a brief screening tool introduced into the MS Society Rehabilitation Services in 1995. This tool has worked very well within Occupational Therapy, but only after certain procedures and processes were established to properly prepare our clients for the overt assessment of their cognition. The tool itself has its inherent strengths and weaknesses. We will discuss this new screening tool and the procedures we have found most helpful in allowing its practical use within our rehabilitation setting.
\end{abstract}

\section{Is the Diagnosis of Multiple Sclerosis a Traumatic Experience? A Discussion of Assessment and Treatment Issues}

Gary Fulcher

Multiple Sclerosis Society of NSW, PO Box 210, Lidcombe, NSW 1825

\begin{abstract}
$\mathrm{M}$ ultiple Sclerosis is a chronic, incurable, potentially disabling disease of the nervous system. In the general population there is little knowledge or understanding of this condition and what does exist is usually incorrect and focused towards the severe sequelae. Being given a diagnosis of MS, therefore, has the potential to be a traumatic experience. The author,
\end{abstract}


having extensive experience in treating trauma-related psychological disorders, noticed MS clients using similar language to those with other trauma experiences. He began to formerly assess the trauma component of clients' presentations and discovered high scores for trauma symptoms on the psychometric questionnaire commonly used to assess post traumatic stress disorder. He then placed an adapted version of the questionnaire in the magazine for people with MS in NSW and asked all recipients to complete it with regard to their own diagnosis and to return it to him. A low return rate occurred but among those who did respond, a surprisingly high average score was found, and approximately one-third of respondents registered scores indicating moderate-to-severe trauma responses. This finding suggests 1) significant directions for assessment and for treatment of clients with established diagnoses of MS, and 2) important directions for practitioners who actually make the diagnosis and for MS-support staff who follow-up the newly diagnosed in the future. The results of the study and the implications and directions for clinicians will be discussed.

\section{"Episodic Stress Responses Model" of Coping with Loss of Ability - Encouraging Application within the MS Population}

Laudy Germanos, Robyn Faine and Daphne Hewson

MS Society of NSW, PO Box 210, Lidcombe, NSW 1825

The experience of coping with multiple sclerosis is often described in relation to traditional grief models. The Episodic Stress Responses Model has been proposed as a more appropriate conceptual model for coping with loss of ability and the changing demands in chronic illness (Hewson 1997). In collaboration with Dr Daphne Hewson, people with MS and their families, the MS Society of NSW has been exploring the applicability of the Episodic Stress Response Model to the experience of living with multiple sclerosis. In this paper the Episodic Stress Responses Model will be reviewed and emerging themes from a qualitative research study will be presented. Participants are invited to join in a brief discussion on the implications of the research themes for clinical practice and for understanding the impact of living with long term illness.

\section{KEYNOTE ADDRESS}

\section{The Syndrome of Developmental Amnesia: Dissociation in Cognitve Memory}

Faraneh Vargha-Khadem

Cognitive Neuroscience Unit, Institute of Child Health, University College

London Medical School \& Great Ormond Hospital for Children, London, UK

The dearth of reports documenting amnesia in children has led to the notion that when damage to the medial temporal lobe system occurs during childhood, the compensatory capacity of the immature brain rescues memory functions. An alternative explanation is that such damage so interferes with the development of learning and memory that it results not in selective impairments but in general mental retardation. Data are presented to counter both of these arguments. Results obtained from a series of amnesic patients with a history of hypoxic ischaemic damage sustained perinatally or during the first year of life indicate a pronounced dissociation between episodic memory, which is severely impaired, and semantic 
memory, which is relatively spared. A second dissociation in these patients is characterised by impaired recall and preserved recognition. These results are discussed in terms of the neuropathology associated with hypoxic ischaemic damage and a hierarchical model of cognitive memory.

\title{
SYMPOSIUM 3: ADVANCES IN PAEDIATRIC ASSESSMENT:
} IMPLICATIONS FOR CLINICAL POPULATIONS

\section{The Nine Box Maze Test-Child Version: A Measure of Non-Verbal Memory}

\author{
Linda Pentland, Vicki Anderson, Sherelle Dye and Stephen Wood \\ Department of Psychology, Royal Children's Hospital, Flemington Road, Parkville, VIC 3052
}

\begin{abstract}
Considerable debate exists around what 'non-verbal' memory constitutes $C$ and how best to measure it. 'Non-verbal' memory is considered to be a multifaceted construct that includes memory for information that is both dependent on the individuals perspective and independent thereof. The former egocentric relations are tapped by traditional neuropsychological measures, whereas the later allocentric associations have largely been overlooked. Allocentric relationships are important in the real-world as these associations allow for the formation of larger scale 'maps' that support navigation. This paper introduces a measure of allocentric memory (Nine Box Maze Test; Abrahams, Pickering, Polkey, \& Morris, 1996) which has recently been modified to be suitable for children. The test will be described and pilot data from a normative sample $(n=60)$ will be presented. This test has been found to be sensitive to development of these skills in children aged 5-12. The application of this test to a clinical research project involving children with circumscribed temporal lobe pathology will also be described.
\end{abstract}

\section{The Contingency Naming Test: A New Test of Cognitive Flexibility?}

Peter Anderson

Academic and Research Coordinator, Department of Psychology,

Royal Children's Hospital, Flemington Road, Parkville, VIC 3052

The Contingency Naming Test (CNT: Taylor et al., 1987) taps higher levels of memory and attentional skills, in particular selective attention, working memory and cognitive flexibility. It differs from most measures of executive function as it was specifically designed for paediatric populations. The CNT involves four subtests, each one requiring a "conceptual shift" and a gradual increment in difficulty level. The CNT incorporates elements from the Stroop Test (Stroop, 1935), however it is not confounded by reading ability. Also, the extra complexity of the CNT enables a more extensive assessment of cognitive flexibility. Currently, it is not used widely as only limited normative data has been available, and its reliability and validity have not been established. This presentation will provide a description of the CNT and the variables that it generates. The sensitivity and specificity of the CNT will be examined using clinical and normative data collected at the Royal Children's Hospital. 


\section{Attentional Skills in the Acute Phase Following Paediatric Traumatic Brain Injury}

Cathy Catroppa and Vicki Anderson

Department of Psychology, Royal Children's Hospital, Flemington Road, Parkville, VIC 3052

$\mathrm{O}$ nly a limited number of studies have investigated attention following pediatric head-injury. The present study examined sustained attention and processing speed in a group of children who had sustained a mild $(n=$ $27)$, moderate $(n=33)$ or severe $(n=16)$ traumatic brain injury (TBI). No significant differences were evident between the TBI groups on reaction time measures. Results did show that the severe TBI group exhibited greater deficits in the area of sustained attention, in comparison to children with mild and moderate injuries, in the acute stage following traumatic brain injury. This difficulty may impact on the future development of skills dependent on intact attentional capacity.

\section{Concept Generation Test as a Measure of \\ Executive Functions in Children - Initial Impressions}

Rani Jacobs, Vicki Anderson and A. Simon Harvey

Department of Psychology, Royal Children's Hospital, Flemington Road, Parkville, VIC 3052

- xecutive functions are difficult to quantify in paediatric populations due Dto the lack of adequately normed measures available for use with children, and the multi-dimensional nature of such tasks, which necessarily incorporate a range of lower level skills. In order to address these issues, a test of conceptual reasoning, the Concept Generation Test (CGT-C) was modified for use with children and administered to 133 children aged between 7.0 and 15.9 years. Developmental trends were noted across age groups with older children using more efficient strategies and achieving higher overall scores. The task was then administered to 30 children with focal frontal lobe injuries involving the pre-frontal cortex and a control group matched for age and sex. The frontal group achieved lower scores than controls when no structure was provided and required more explicit cues to complete the task than controls. Within the frontal group, differences were evident according to lesion lateralisation (right vs left focal lesions).

\section{PAPER SESSION 1: DEGENERATIVE DISORDERS ASSOCIATED WITH AGING}

\section{Longterm Cognitive Sequelae of a Single Stroke Event}

Jacqueline Anderson, V. Srikanth, Michael Saling and Geoff Donnan

University of Melbourne, 35 Power Street, Toorak, VIC 3142

R ecent research has found an increased incidence of dementia in patients who have suffered a single stroke event, relative to the normal population. No comprehensive longitudinal study of cognitive performance has been conducted with this population, however. Using a community based population of first ever stroke patients obtained from the North Eastern Melbourne Stroke Incidence Study (NEMESIS), we examined the cognitive profile of 80 patients at three and 12 months post-stroke and compared their performance to pre-stroke cognitive functioning. The resultant findings detail the nature and extent of cognitive change developed after a single stroke during the first 12 months. 


\title{
Cognitive Changes in Parkinson's Disease: Evidence from Patients' Subjective Complaints and Reported Day to Day Functioning
}

\author{
Felicity Whitworth, B. Kirkwood, C. Marigliani, R. lansek and R. Bell \\ Kingston Centre/University of Melbourne, Kingston Centre, Warrigal Road, Cheltenham, VIC 3192
}

\begin{abstract}
$\mathrm{N}^{2}$ europsychological and experimental investigations of people with Parkinson's disease (PD) have revealed a range of cognitive impairments that can be demonstrated even early in the course of the disease. It is not yet clear, however, what is the significance of the various reported neuropsychological impairments at a clinical level. Clinically, many patients with PD complain of experiences such as confusion and forgetfulness, which may be associated with symptoms such as depression, anxiety or fatigue, as well as with difficulties in day to day functioning. Furthermore, it appears that these difficulties may be ameliorated or avoided to some degree through the development of appropriate strategies. In order for further understanding of the neuropsychological sequelae of PD and development of appropriate rehabilitation approaches, there is a need for systematic documentation of the clinical manifestation of neuropsychological deficits. This paper presents the results of an in-depth, multidisciplinary examination of neuropsychological subjective complaints, along with associated difficulties in day-to-day activities and communication, in a group of eight patients with PD. Also reported are the results of neuropsychological and communication tests. Patients' subjective complaints were documented using a grounded theory approach, in which expectations of findings are minimised. The results indicate that patients may complain of a number of subjective changes and functional difficulties, with some complaints being more consistent than others. Marked effects on day to day functioning were reported even in the absence of significant deficits on testing. Patients vary in the level of their understanding of their difficulties, along with how much they are able to avoid or cope with difficulties when they occur. Clear precipitants and more general psychological consequences are also described. The results provide a basis for the development of a specific tool to systematically investigate the clinically apparent neuropsychological changes in PD.
\end{abstract}

\section{A Neuropsychological Investigation of Dementia in Motor Neurone Disease}

Fiona Barson, Glynda Kinsella, B. Ong and S. Mathers

LaTrobe University, Bethlehem Hospital, Bethleham Hospital, 476 Kooyong Road, Caulfield, VIC 3162

$\mathrm{W}$ ith the identification and clinicopathological description of frontotemporal dementia (FTD) in the 1980's came the recognition that the dementia associated with motor neurone disease (MND) is a subtype of FTD. Diagnostic criteria for FTD have now been published and offer the opportunity for more precise diagnosis of dementia in MND. Prospective neuropsychological studies of MND patients who are at risk of FTD offer a unique opportunity to study the cognitive deficits of this dementia in its earliest stages. Patterns of neuropsychological test performance that relate to the clinical diagnosis of FTD can be described and the impact of these cognitive deficits on everyday living can be ascertained. Such information may assist in the early identification of non-MND presentations of this dementia.

The aims of this study were threefold: 1) to compare different clinical methods of diagnosing dementia in a sample of 69 patients with MND, 2) to determine whether neuropsychological tests could distinguish between 
MND patients with a clinical diagnosis of dementia and those without and 3 ) to determine whether there were a significant relationship between performance on neuropsychological tests and carers' reports of dysexecutive impairment in daily living. The results indicated that when diagnostic criteria for frontotemporal dementia (FTD) were used as part of a questionnaire method of diagnosing dementia, the incidence of dementia in MND was considerably greater than traditional estimates suggest. Through a series of logistic and multiple regressions the results demonstrated that neuropsychological test performance related well to diagnostic classifications of dementia. MND patients with a clinical diagnosis of dementia were likely to demonstrate impaired new learning; poor working memory and planning; slowness in information processing and rigidity in thinking. The finding that neuropsychological impairment correlated with behavioural features of dysexecutive impairment in daily living (as measured by the DEX) suggests that the experience of cognitive deficits in FTD is discernible in everyday activities. Broadening the management focus of MND to address cognitive/behavioural issues will provide additional support for individuals with MND, as well as for families and carers.

\section{Dementia with Lewy Bodies: Current Dilemmas and Future Directions}

Jennifer Bradshaw

Austin and Repatriation Medical Centre, Repatriation Campus, Department Clinical Neuropsychology, Locked Bag 1, West Heidelberg, VIC 3081

ementia with Lewy bodies (DLB) has been heralded as the second most common cause of degenerative dementia in old age. Since the publication of consensus guidelines for the clinical and pathological diagnosis of DLB in 1996, research interest in the condition has grown considerably. With this interest a number of controversies have emerged.

This paper will attempt to provide an overview of the current clinical issues relevant to clinicians attempting to differentiate this condition from other degenerative dementias. Issues to be addressed include - terminology and what is appropriate; use of currently available clinical criteria; diagnostic accuracy; associations with related conditions (Alzheimer's and Parkinson's disease).

A particular emphasis will be placed on reviewing the available neuropsychological literature with respect to DLB. Dilemmas and controversies will be highlighted and illustrated with case material. The paper will conclude with some suggested directions for future research.

\section{Neuropsychological Impairment in Older Adults with Late Onset Huntington's Disease}

Bernadette Bibb and E. McCusker

Department of Medical Psychology, Westmead Hospital, PO Box 533, Westmead, NSW 2145

$\mathrm{H}$ untington's disease (HD) is an autosomal dominant inherited disorder characterised by progressive involuntary choreiform movements, dementia, and personality disturbance. Disease onset, as confirmed by DNA analysis, is most common in the middle adult years, with a disease course spanning 10-20 years. The literature reports that in cases of late onset HD (approximately $25-35 \%$ of cases) cognitive changes are absent or minimal. We present data from patients who presented at the Huntington's Disease 
Clinic at Westmead Hospital that contradict this claim. Fifteen patients aged 55 years and over (55-87 year range) with late onset HD underwent neuropsychological assessment. Cognitive impairment was evident in memory and learning, and executive functions, consistent with striatal degeneration. Functional index ratings, using the Independence Scale, indicated that these older patients maintained a reasonable level of functional independence despite the documented level of cognitive impairments. The findings have implications for long-term management of elderly patients with HD.

\title{
SYMPOSIUM 4: ISSUES IN NEUROPSYCHIATRY
}

\section{Retrograde Memory Loss Following ECT: Fact or Fiction?}

Maggie Phillips and Anne Unkenstein

Melbourne Extended Care and Rehabilitation Service,

North West Hospital, Poplar Road, Parkville, VIC 3052

\begin{abstract}
Cognitive side effects following the administration of ECT have been well documented in the literature ever since this treatment was first introduced for depression in the late 1930's. The most commonly reported disturbance of neuropsychological significance involves memory function, with retrograde memory loss for autobiographical material being especially noted in a number of studies. This paper presents an overview of the literature concerning retrograde memory loss following ECT. Patient and treatment characteristics thought to contribute to the ongoing controversy over the issue of whether ECT causes permanent deficits in retrograde memory will be discussed, with particular attention paid to the relationship between ECT parameters, aging and cognitive impairments. The putative mechanisms thought to be responsible for retrograde memory loss will be considered. Two clinical cases will be described to illustrate the nature, extent and duration of retrograde memory loss following ECT. The importance of detailed assessment, careful history taking and knowledge of the clinical literature to avoid the pitfalls of misdiagnosis of subjective complaints of autobiographical memory impairment will be highlighted.
\end{abstract}

\section{Asperger Syndrome in an Aged Psychiatry Service - A Case Study}

\author{
Elizabeth Mullaly \\ Caulfield General Medical Centre, 260 Kooyong Road, Caulfield
}

\begin{abstract}
$\mathrm{W}$ hile Asperger Syndrome is becoming more well-recognised in paediatric populations less is known about the ongoing effects of the disorder in adult and later life. M.G. is a 65 year old man who has had lifelong contact with mental health services and many diagnoses including schizophrenia, OCD and psychopathic personality disorder. His case was reviewed when he turned 65 and entered an Aged Psychiatry Service. Many features were consistent with Asperger Syndrome, a diagnosis that had previously not been considered. Features of the case will be discussed including the nonverbal learning disability profile that showed up on neuropsychological assessment. The diagnosis assisted in clarifying management approaches and goal-setting for the treating team.
\end{abstract}




\section{Capgras and its Friends and Relations}

Nora Breen and Max Coltheart

case of CAPGRAS is currently being investigated. This case seems to
ders and assuch provides interesting insights into the nature of this disorder.

SYMPOSIUM 5: CHILDHOOD EPILEPSIES

\section{Difficulties Encountered with Prediction of Cognitive Outcome in Children who Undergo Surgery for Epilepsy}

Suncia Sunny Lah

Sydney Children's Hospital, Level o North, High Street, Randwick, NSW 2031

$\mathrm{R}$ eliable prediction of cognitive outcome is the ultimate aim of neuropsychological evaluation prior to epilepsy surgery. In adults, risk of verbal memory impairment post left temporal lobectomy is well recognised. In children, interest and availability of surgical treatment for epilepsy has gradually increased over the years. However, there is a dearth of either clinical or research papers dealing with cognitive and behavioural outcome in children who undergo surgery. At present paediatric neuropsychologists working in the area of epilepsy utilise clinical practices and knowledge from adult literature. However, children who are potential candidates for epilepsy surgery vary significantly in their developmental and chronological ages. Furthermore, a considerable number of patients undergo extratemporal surgery. All these factors influence the manner, scope and means of examinations as well as predictions of outcome.

I will discuss difficulties involved in conducting presurgical evaluation of a child, using an invasive intracarotid sodium amobarbital (ISA) procedure and some of the ways to overcome them. A video of a child who underwent the ISA procedure will be presented.

Review of 13 children aged 1-18 years (median 12 years) who underwent temporal $(N=9)$ and extratemporal $(N=4)$ focal resections for intractable epilepsy showed that there was either no change or small improvements in overall IQ in 12 of the 13 patients (Lah, et al, 1999). In verbal learning, a significant decline was noticed in 4 out of 11 patients, with 2 patients having an extratemporal resection. Decline in verbal learning was independent of changes in IQ or laterality of resection.

To conclude, prediction of cognitive outcome in children who undergo surgery for epilepsy is limited by the following: surgery often involving young and/or developmentally delayed children, more frequent extratemporal resections, research suggesting that immediate cognitive risks may differ from those encountered by adults as well as a paucity of research data. 


\section{A Comparison of Landau-Kleffner Syndrome and Continuous Spike and Wave Syndrome. Case Studies}

Anna Mandalis

Sydney Children's Hospital, Level o North, High Street, Randwick, NSW 2031

T andau-Kleffner Syndrome (LKS) and Continuous Spike and Wave LSyndrome (CSWS) are paediatric-specific epilepsy disorders. In both cases the epilepsy is usually severe and intractable and the most common treatment alternative to anti-convulsant medication is steroids. The two syndromes are usually differentiated by the pattern of EEG findings. Although the initial presentation of children with LKS and CSWS can be similar in that they usually have significant language and behavioural deficits, a significant auditory agnosia is commonly observed in children with LKS. The presentation will compare and contrast a case of LKS with a case of CSWS. Both children were neuropsychologically assessed pre- and post-steroid treatment. Footage of the child with LKS pre and post steroid treatment will also be shown. The neuropsychological assessment results and video footage will illustrate the impact and benefits of steroid treatment. The longterm outcome of children with these epilepsy syndromes will be discussed.

\section{Neuropsychological Features of Different Childhood Epilepsy Syndromes}

Antoinette Redoblado

Sydney Children's Hospital, Level o North, High Street, Randwick, NSW 2031

$\mathrm{I}^{\mathrm{n}}$ n adults, different epilepsy syndromes are associated with unique neuropsychological features. Temporal lobe epilepsy (TLE) is associated with memory impairment. Frontal lobe epilepsy (FLE) is associated with executive deficits. Compared to focal epilepsy syndromes, generalised epilepsy is associated with more widespread cognitive deficits. However, there is minimal information on the neuropsychological features of different epilepsy syndromes in children.

We conducted a study aimed at delineating the neuropsychological features of common epilepsy syndromes in children. Sixty-four subjects with severe epilepsy were recruited from either Sydney Children's Hospital or The New Children's Hospital to participate in this study. There were three groups of subjects: 22 FLE, 21 TLE and 21 generalised epilepsy. All subjects were given a battery of standardised and developmentally-normed neuropsychological tests measuring intelligence, memory and executive function. All subjects showed low average IQ. Contrary to adult findings children with TLE did not show material-specific memory impairment. Syndrome-specific cognitive deficits were only found in the FLE group. These children showed specific impairments in executive skills such as planning and verbal inhibition. The results of this study provide invaluable information about the unique impairments and needs associated with individual epilepsy syndromes which can be used to create treatment plans and enhance the validity of pre-surgical evaluation. 


\title{
PAPER SESSION 2: DEVELOPMENTAL NEUROPSYCHOLOGY: CLINICAL AND RESEARCH PERSPECTIVES
}

\section{Callosal Agenesis Down the Track - A Case Study}

Catherine Willmott

Epworth Hospital, Bethesda Rehabilitation Centre, c/o Psychology Department, 89 Bridge Road, Richmond, VIC 3121

This paper forms a discussion of a history of callosal agenesis in a twenty 1 year old girl which was only recently diagnosed following a traumatic brain injury. It will address issues of the patient's social and academic history and development in light of "plasticity" theories. A review of means available to test the function of the corpus callosum will be covered and some of this assessment will be shown on video. Rehabilitation directives for this case will be outlined and future considerations regarding traumatic brain injury recovery in such a complex case will be discussed.

\section{Verbal Deficits in the Syndrome of Nonverbal Learning Disabilities: Comparing the Narrative Discourse of Children with Asperger Syndrome and Early Hydrocephalus}

Bridgit Ryburn, Vicki Anderson and Roger Wales

University of Melbourne, 637 Park Street, Brunswick, VIC 3056

\begin{abstract}
Tt has recently been suggested by Rourke that a variety of disorders such 1 as Asperger Syndrome and Early Hydrocephalus can be usefully represented by the broader category of the Syndrome of Nonverbal Learning Disabilities. Although basic linguistic skills such as pronunciation, grammar and vocabulary are described as relatively intact in children with this primarily nonverbal disability, pragmatic skills are postulated as a substantial area of weakness. The aim of this study was to empirically investigate the language of these children at several levels using a narrative discourse task, including both linguistic and more pragmatic analyses of information structure. A comprehensive neuropsychological battery was also administered. Three groups of children of average intelligence, between the ages of 8 and 14 were included in the study; 1) 10 children with uncomplicated Early Hydrocephalus, 2) 16 children with a diagnosis of Asperger Syndrome and 3) 20 healthy normal control subjects. The findings from the discourse task indicate different profiles for the two clinical groups. The results and their implication for the theory of nonverbal learning disability will be discussed.
\end{abstract}

\section{Specific Deficits in Attention for Attention Deficit/Hyperactivity Disorder Using the Task-Set Switching Paradigm}

Kitty Wu, Vicki Anderson and Umbersto Castiello

Psychology Department, University of Melbourne, School of Behavioural Science, 12/F, Redmond Barry Building, University of Melbourne, Parkville, VIC 3052

The present study investigates the deficits of children with Attention Deficit/Hyperactivity Disorder (AD/HD) in various aspects of attention. The rationale of the study is based on recent neurophysiological and neuropsychological studies that suggest frontal lobes dysfunction in AD/HD. As frontal lobe functioning is closely related to attention, $\mathrm{AD} / \mathrm{HD}$ is hypoth- 
esised to be associated with specific deficits in attention. Participants were 81 children with AD/HD and 29 age-matched controls. Standardised neuropsychological tests and experimental conditions based on the task-set switching paradigm were utilised for measuring different aspects of attention. In the experiment, subjects have to switch between the colour-naming and word-reading tasks on every 2 nd trial. Neutral and Stroop stimuli were presented in separate blocks of trials. Within this alternating runs paradigm, negative priming effect was manipulated for the word-reading task in the condition with Stroop stimuli. In the baseline conditions, no switching was required. Performances (i.e. accuracy, speed and variability of reaction time) in the alternating runs paradigm were compared with those in the baseline conditions. Results from experiments support the hypotheses that $\mathrm{AD} / \mathrm{HD}$ is associated with specific deficits in speed of processing and resistance for the interference of Stroop effect i.e. selective attention. The comorbidity of LD is related to deficits in switching attention and resistance for negative priming effect. Findings from standardised neuropsychological tests are consistent with the results of the experiments. In addition, the deficit associated with AD/HD in sustained attention is identified in tests. Results are discussed in terms of current theories in task-set switching paradigm and the specific deficits of $\mathrm{AD} / \mathrm{HD}$ and the co-morbidity of LD.

\section{Understanding and Addressing the Needs of Siblings of Children with Acquired Brain Injury}

\section{Samantha Burnsnall}

Griffith University (Assistance Mater Children's), Centre for

Human Services, Griffith University, Logan Campus, Nathan, QLD 4111

With advances in medical technology more children are surviving acquired brain injury (ABI) that may otherwise have been fatal. Statistics suggest that approximately 220 in every 100000 children are affected by head injury (Snow \& Hooper, 1994). Although these statistics are high, they do not convey the full magnitude of the problem, since ABI is an issue impacting on the entire family unit. Although research examining the impact of $\mathrm{ABI}$ on the family is growing, little research has examined the impact that a child's acquired brain injury may have on his/her siblings, the pre-existing sibling relationship and the consequent adjustment of these children. This study examined the impact a child's ABI has on siblings. In conjunction with psychosocial outcome measures, siblings of children with ABI were interviewed about the perceived impact their brother or sister's injury had on different areas of their lives. Preliminary findings of this initial study reveal that siblings perceive their brother or sister's injury/illness to be the central cause of many negative and positive changes within different domains of their lives. The findings suggest that siblings experience an extremely complex process that is not recognised in the current rehabilitation continuum. Sibling responses suggested several strategies for addressing their particular needs at different phases of their brother or sister's rehabilitation process. The development and implementation of appropriate intervention and service delivery strategies are outlined and the difficulties of addressing the needs of this target group are highlighted. The importance of adopting innovative and timely intervention strategies to this population is discussed. 


\title{
Long-Term Memory in a Learning Disorders Clinic Cohort
}

Wayne Levick

John Hunter Children's Hospital, Locked Bag 1, Hunter Region Mail Centre, NSW 2310

\begin{abstract}
A deficit in the ability to store new verbal and non-verbal information in long-term memory is the main feature of the amnesic syndrome described in adults and children with medial temporal and diencephalic dysfunction. In recent years a number of cases have been reported suggesting that children and adults of average IQ and without apparent acquired brain damage can display varying degrees of deficit in the ability to store new information in long-term memory. The deficit typically shows up on testing as poor initial learning and/or delayed recall. This study examined the incidence of this type of memory deficit in a group of 233 children referred for assessment of specific learning difficulties. Each child was administered a selection of subtests of the WRAML in addition to IQ and reading, spelling and arithmetic measures. Performance on the learning trials and at delayed recall on the WRAML. Verbal and Visual Learning subtests was examined. The criteria for deficit was set as a scaled score of 6 or less and/or a delayed recall savings score more than $25 \%$ worse than that for the appropriate age group in the WRAML normative sample on both subtests. Using these criteria, 12 children $(5.2 \%$ of the sample) were identified as having a deficit in verbal and non-verbal long-term memory. This group did not differ from the rest of the Clinic group in age, PIQ, verbal memory span, reading, spelling, or arithmetic. The theoretical and clinical implications are briefly considered.
\end{abstract}

\section{Performance of Children and Adolescents with TBI on Prospective Memory Tasks}

Roberta E Dobson-Patterson, D. Shum, L. Blowers and G. Wallace

School of Applied Psychology, Griffith University, Mt Gravatt Campus, Nathan, QLD 4111

Drospective memory, or the ability to remember to perform planned actions, is considered to be important and relevant for independent functioning in individuals with traumatic brain injury (TBI). This type of memory has been found to be impaired in adults with TBI (e.g., Shum, Valentine \& Cutmore, 1999), but few studies have been conducted to examine the effects of TBI on this type of memory in children. This study employed a dual-task paradigm to compare the performance of 13 children (age range $=8$ to 15 years old) with moderate to severe TBI to 13 controls (matched for age and gender) on time- and event-based prospective memory tasks. In this paradigm, the ongoing task required participants to answer a series of age-appropriate trivia questions by pressing one of three keys ( $a, b$, or c) for about 15 minutes. In the time-based condition, participants had to ring a bell and report their level of performance (as shown on the computer screen) on the ongoing task every three minutes. In the event-based condition, they had to perform the action every time they saw a target word (e.g., Australia) appear in a trivia question. A total of five responses were required in each condition. Children in the TBI group were found to perform significantly worse than controls in both time- and event-based conditions. However, unlike the results reported by Shum, et al., (1999), children in the TBI group did not show the characteristic time-versus event-based dissociation. Neither time estimation ability nor retrospective memory perfor- 
mance could adequately explain the impairment of the TBI group on the two prospective memory tasks. These results suggested that TBI might have a different effect on prospective memory for children than for adults, and further investigations are needed to ascertain the reason for this difference.

\title{
PAPER SESSION 3: APPROACHES TO ASSESSMENT ACROSS THE LIFESPAN
}

\section{Problems and Pitfalls in the Assessment of Posttraumatic Amnesia: A Critical Review}

\author{
Robyn Tate and Anne Pfaff \\ Rehabilitation Studies Unit, Department of Medicine, University of Sydney, \\ Royal Rehabilitation Centre, Sydney, PO Box 6, Ryde, NSW 1680
}

\begin{abstract}
$\mathrm{F}$ ollowing emergence from coma, patients with traumatic brain injury usually experience a period of posttraumatic amnesia (PTA) in which they are confused, amnesic for ongoing events, and frequently behaviourally agitated. Determining the duration of PTA is important: it is a reliable index of the severity of the injury, is one of the best indicators of the ultimate level of recovery and outcome, and many clinical management decisions are made at the time the patient is considered to have emerged from PTA. Although a variety of procedures and instruments are available to measure the duration of PTA, their applications in clinical practice are far from ideal and raise many validity issues. There are inconsistencies in the literature, with respect to the frequency with which PTA should be examined, which measure should be used, and how to validly determine when PTA has ended. Moreover, PTA testing in rehabilitation units can continue for many months, and it is not uncommon for the patient's relatives to anxiously grasp onto the concept of PTA, including tutoring the patient in readiness for PTA testing in an effort to expedite their emergence from PTA. All these difficulties pose questions about the role and conduct of PTA testing. The paper illustrates these and other problems and pitfalls in the measurement of PTA, drawing upon the data from our four-year study of 148 patients who were examined on a daily basis until they emerged from PTA, and provides suggestions for improved clinical practice.
\end{abstract}

\section{Timed Up-And-Go Test in Children: What Does It Tell Us?}

Elizabeth Williams, S. Carroll and M. Galea

School of Physiotherapy, University of Melbourne, VIC 3010

The Timed "Up and Go" or TUG Test, was selected for this study because it has been shown to be a useful and practical objective measure of physical mobility in elderly people, reliable between raters and over time. However for the paediatric population it reflects a complex, coordinated functional activity requiring motor planning, initiation, motor control and intact sensory systems. The test is to stand from sit, walk 3 meters, turn and return to the seat, a fundamental skill at work, home or school. Subjects were 113 children between 3 and 9 years, boys $(n=50)$ and girls $(n=40)$. They were studied over 6 months in three age groups to reflect the immature, the developing and the mature child in respect of motor skills. Results showed no significant difference between males and females with males scoring an average of 5.5 and females 5.6 seconds for the task. Younger children, were slower, more variable and showed the most change over 5 
months than older children. Average scores for 3-4 years $=6.8$ seconds (SD $1.4), 5-7$ years $=5.5$ seconds (SD 1.3), and 7-9 years 4.9 seconds (SD 1.0). Comparison of time points at 0 and five months showed a statistically significant improvement of 1.1 seconds for the 3-4 year age group, only 0.3 seconds in the 5-6 year olds and children of 7-9 years were stable. The TUG Test is easy to perform and appears a sensitive, objective test of mobility in the paediatric population. It may be useful as a standard measure in ambulatory children with neurological disorders. Current studies are being undertaken to examine the efficacy of this test in an ambulatory disabled population.

\section{The Minimally Responsive Client: A Partnership Approach to Assessment in Traumatic Brain Injury}

Christine Wallace, Michele Lammi, Vanessa Smith and Robyn Tate

Royal Rehabilitation Centre, Sydney, PO Box 6, Ryde, NSW 1680

The minimally responsive client in the subacute rehabilitation setting, is often difficult to objectively assess. In recognising the expertise of Occupational Therapists and Speech Pathologists, we adopted a partnership approach in an attempt to more comprehensively and reliably identify cognitive and sensory changes in this client group.

The Western Neuro Sensory Stimulation Profile (WNSSP) was chosen as the collaborative assessment tool. It consists of nine subscales, including Arousal/Attention (AA), Auditory Response (AR), Auditory Comprehension (AC), Expressive Communication (EC), Visual Tracking (VT), Visual Comprehension (VC), Tactile Response (TR), Object Manipulation (OM) and Olfactory Response (OR). It is designed to assess and monitor changes in cognitive function in 'slow-to-recover' traumatic brain injury clients. Total scores range from $0-113$. Higher scores indicate better levels of functioning.

Over the last two years, a group of 25 clients (15\% of all admissions to the Brain Injury Unit) were administered the WNSSP. Research was undertaken using retrospective data collection from client files, to examine whether the WNSSP is effective in showing change in a subacute population.

A subset of 12, who were administered the WNSSP on at least four occasions, were studied in detail. The initial score on the WNSSP was on average 28 and on admission their Functional Independence Measure (FIM) score was 18 in each case. Testing commenced on average at 50 days post trauma. Although the mean scores increased from an average of 28 to 42 between the first and fourth test occasions, the difference was not statistically significant. Significant differences were found however, on particular subscales. These comprised OM and VT and a trend to significance was demonstrated for AR. These results suggest that cognitive and sensory functions subserved by these scales are probably the first to emerge in the minimally responsive client and should be targeted early in the intervention phase.

\section{Age-Effects in Prospective Remembering Using a Prose-Reading Task}

Glynda Kinsella, B. Ong and R. Hester

La Trobe University, School of Psychological Science, Bundoora, VIC 3083 
The most frequently reported errors of memory in daily life are those 1 which involve forgetting to do things, or prospective remembering (ProR). Event-based ProR refers to situations where an environmental event or cue signals the timeliness for executing the intended action. An accurate account of the effects of normal aging in ProR is essential to provide a standard against which pathological changes can be assessed. Age-differences have been reported for event-based ProR tasks where working memory demands are high. However, these results have been based on laboratorybased methodologies and naturalistic studies have not identified the same age-related decline. Furthermore, methodological limitations of many laboratory-based tasks of ProR have limited the interpretation of the research.

The present study was undertaken to investigate the potential for age-related changes in ProR by using a recently developed, and easily administered, quasi-naturalistic task to overcome some of the limitations identified with the existing laboratory-based methodologies. The relationships between ProR task performance, self-report of ProR and neuropsychological measures of individual differences were also explored. The sample consisted of 30 younger and 30 older adults. Results showed that when event-based ProR is assessed in low demand working memory conditions, older adults can perform as well as younger adults. Interestingly, there was no relationship between self-report and performance on the quasi-naturalistic ProR task, and the use and interpretation of self-report of memory performance will be discussed. The found intercorrelations between performance on ProR and neuropsychological measures of individual differences will be evaluated within a model of working memory resources. The advantages and disadvantages of the study methodology for clinical neuropsychological assessment of ProR and aging will be considered.

\section{Anterior Attentional Functions in Mild Alzheimer's Disease}

Maria Hennessy and S. Mintarti

James Cook University, School of Psychology, James Cook University, Townsville, QLD 4811

The assessment of attention in the early stages of Alzheimer's disease

(AD) is relatively under-researched and often neglected in clinical practice. Stuss et al's (1995) model of anterior attentional functions was used to select a number of neuropsychological tasks each measuring hypothesised components of attention. These tasks were administered to 17 mild AD individuals and 22 controls. Different patterns of impairment on specific attentional components were found. Sustaining attention on demanding tasks, sharing, suppressing and switching attention were found to be impaired. However sustaining attention on tasks with low levels of demand, concentrating and preparatory attention were intact in mild AD. This differential pattern of neuropsychological impairment has important implications for early detection, and the refinement of neurocognitive models of attention.

\section{What Did I Have to Do Again? The Relationship Between Neuropsychological Measures and Everyday Prospective Memory in TBI}

Jilianne Kinch and Skye McDonald

he ability to remember to perform an intended action sometime in the
future is a fundamental aspect of everyday functioning, yet it also forms 
one of the most common and annoying forms of memory failure. This present study investigated the effect of severe traumatic brain injury (TBI) on prospective memory. In addition, this study also sought to understand the mechanisms by which one remembers future actions, namely executive functioning and retrospective memory. Thirteen patients with severe TBI and 13 matched control subjects were assessed on two novel, yet naturalistic experimental measures of prospective memory and standard tests of neuropsychological functioning. As expected those in the TBI group performed worse than the Non-TBI group on standard retrospective memory tasks, and importantly also showed poorer performance on a time-based prospective memory task. These findings indicated that TBI affected not only retrospective but also prospective memory functioning. The effect of TBI on memory was investigated further, by examining the relative contribution of executive functioning and retrospective memory to prospective memory performance. Interestingly, results from multiple regression analyses suggested that performance on time-based prospective memory tasks (such as remembering the time of a doctor's appointment) is heavily reliant upon executive functioning (rather than retrospective memory). In addition, successful completion of event-based prospective memory tasks (for example, remembering to post a letter in the presence of a post box) was primarily attributable to retrospective memory. Hence, the results suggest that whilst prospective memory requires both executive function and retrospective memory, it appears that the ability to remember to do something at a particular time is dependent mostly upon executive functioning, whereas, the actual content of this memory is reliant upon retrospective memory. Implications of these findings for the rehabilitation of prospective memory impairment in individuals with TBI are discussed.

\section{PAPER SESSION 4A: RISK TAKING BEHAVIOUR: NEUROBEHAVIOURAL IMPLICATIONS}

\section{Hypoxic Brain Injury: Can Pharmacotherapy Alter Functional Outcome?}

Joan Tierney

Brain Disorders Program, ARMC, PO Box 269, Kew, VIC 3101

Wypoxic brain injury is generally considered to have a poorer outcome than traumatic brain injury. Patients who do not make rapid recovery in the first few months post injury are often deemed to be unsuitable for further rehabilitation input.

This paper presents two case studies of young men who had been hospitalised for 9 and 2 years respectively in longstay psychiatric wards. Both had survived suicide attempts by hanging. Each had failed previous attempts at $\mathrm{ABI}$ rehabilitation prior to long term institutionalisation. Numerous psychiatric interventions including trials of antipsychotic and antidepressant medication and numerous behavioural programs had been unsuccessful in improving their level of function. Both remained grossly adynamic, poorly mobile and with minimal communication skills.

Significant functional improvement was noted in both cases on a combination of a selective serotonin reuptake inhibitor, and dopamine agonists. Both were noted to deteriorate in functional abilities when the dosage of any one of three agents in this combination was reduced, and improved with restitu- 
tion of the dosage. Both of these young men were subsequently discharged to supported community accommodation and continue to make gains more than 3 years post discharge.

The role of various neurotransmitters in normal brain function is now recognised. The effect of brain injury on these neurotransmitter systems, however, is still poorly understood. The possible role of serotonergic and dopaminergic neurotransmitter systems in recovery from ABI, and the therapeutic implications will be explored.

\title{
Heroin Overdose and Hypoxia: Implications for Rehabilitation
}

Jenny Todd and Trish O'Brien

Royal Talbot Rehabilitation Centre, ABI Unit, Yarra Boulevard, Kew, VIC 3101

Tn recent years the incidence of heroin overdose has steadily risen and the Iincrease in overdose related deaths is well documented. Less publicised is the significant disability that can result from hypoxic brain injury following heroin overdose. Over the past 2 to 3 years staff at the ABI Unit at Royal Talbot Rehabilitation Centre have noted an increase in referrals post overdose. While this group is varied in terms of type and level of disability, a pattern emerged with memory abilities appearing to be relatively better preserved than was observed in other hypoxic patients. To further examine this group a retrospective study was conducted of hypoxic admissions to Royal Talbot between June 1997 and June 1999. The group consisted of 26 hypoxic patients, aged 18 to 59 with the following causes of hypoxia: cardiac arrest $(n=11)$, drug overdose $(n=10)$, attempted hanging $(n=4)$, and seizure $(n=1)$. Heroin was listed as the main substance in 8 of the drug overdose cases with the other case being listed as a poly drug overdose. The results indicated significant levels of disability in the hypoxia group as a whole with 22 requiring supported accommodation or extensive family help and/or other services to live in the community. In terms of specific deficits the results did suggest a subgroup within the drug overdose group with relatively better preserved memory though severe memory deficits also occurred in some individuals. In reviewing the data a number of complicating factors within the heroin group were apparent. The vulnerability of this group was highlighted by the fact that over the two year period 2 of the 9 drug overdose group had died from a subsequent overdose. From a rehabilitation services perspective a key issue which emerged was the combination of an ABI and drug use which made it difficult for either ABI or Drug \& Alcohol services to adequately meet the needs of these individuals. This paper will discuss the challenges that rehabilitation services should address when dealing with this group.

\section{Language Learning Disabilities and Risk for Substance Abuse: Exploring Connections}

\author{
Pamela Snow \\ Deakin University, School of Psychology, 221 Burwood Highway, Burwood, VIC 3125
}

I arge volumes of recent research efforts have been devoted to the identiLfication of risk and protective factors with respect to young people and drug-related harm. There is a substantial body of evidence attesting to the link between a range of specific developmental disorders (e.g., attentiondeficit/hyperactivity disorder $[\mathrm{AD} / \mathrm{HD}]$ and learning disability) on the one 
hand, and adolescent substance abuse on the other, particularly in those individuals who also display conduct disorders. A range of cognitive/executive function disturbances have been described in children and adolescents with learning and/or attentional disorders. These include poor planning and organisation, poor self monitoring, reduced impulse control, difficulties sustaining attention and concentration, and problems abstracting information across contexts. Such deficits may be antecedents, concomitants and/or effects of drug use, and the presence of conduct disorder is frequently a significant moderator variable in this equation. Very little research has been conducted, however, into the role of language learning deficits in the lives of young people who are academically and/or socially marginalised and likely to experiment with licit and illicit drugs. This reflects in part an historical lack of multidisciplinary research into these populations. The aim of this paper will be to review the recent, but compelling evidence which suggests that clinicians working with young people with language disorders need to be aware of their elevated risk for substance abuse - and conversely, workers in the drug and alcohol field need to have a better understanding about the implications of developmental language learning disability for therapeutic interventions. Recent research evidence from the speech pathology, neuropsychology and drug and alcohol fields will be integrated in this paper, and implications for research and clinical practice will be identified.

\section{PAPER SESSION 4B: PSYCHOSOCIAL ADJUSTMENT OF CHILDREN FOLLOWING TRAUMATIC BRAIN INJURY}

\section{Psychosocial Adjustment of Children Following Traumatic Brain Injury}

Tim Hannan, Kath Bakker and R. Brunsdon

New Children's Hospital, Westmead, NSW 2145

$\mathrm{I}_{\mathrm{i}}^{\mathrm{t}}$ $t$ is well known that the child who suffers a traumatic brain injury (TBI) is at an increased risk of experiencing a range of short-term and/or enduring psychosocial difficulties. These problems may either arise anew as a direct result of the brain injury, represent an exacerbation of premorbid difficulties, or emerge as a reaction to the perceived effects of the injury on cognitive or physical functioning. While recent research has advanced our understanding of the nature and prevalence of psychosocial difficulties that commonly follow TBI in children, many clinically important questions remain unanswered. In this session, the presenters will discuss research examining different aspects of psychosocial adjustment after TBI, and consider the implications for clinical practice in the rehabilitation of the braininjured child. Each paper includes a discussion of the theoretical and methodological issues that complicate research in this domain.

\section{Social Functioning Following Traumatic Brain Injury in Children}

N Reynolds, T. Hannan, D. Erickson, R. Brunsdon and K. Bakker

University of Sydney

While it is recognised that children who have suffered a traumatic brain injury (TBI) appear to be at greater risk for difficulties in social interaction with peers, the nature and severity of such difficulties have not been well investigated. The aim of the present study was to examine detailed 
information from multiple informants on the social performance of children following TBI. The experimental group consisted of sixteen children aged 7 to 12 years who had suffered a moderate to severe brain injury one to five years previously. Children's social performance was conceptualised as involving social skills, social cognition and behavioural self-control. Children with TBI performed significantly poorer than controls on the social performance, social skills and social cognition measures. In addition, children with TBI identified themselves as experiencing the same social skill difficulties as reported by their parents and teachers. Based on the findings of this study, further development into treatment recommendations, therapeutic interventions and family education should be conducted. It is expected that the results from this study will help to refine and develop psychosocial treatment programs to help children recover from a TBI.

\title{
Post Traumatic Stress Disorder in Children Following Road Traffic Accidents
}

Fiona Mather, Robyn Tate and Tim Hannan

University of Sydney

$\mathrm{T}$ his project investigated the frequency, course and comorbidity of posttraumatic stress disorder (PTSD) in children following road traffic accidents (RTA). Forty-three children and their parents were interviewed six weeks following an RTA. A subset $(n=32)$ was re-interviewed seven weeks later. Initially, 74\% of children reported significant PTSD symptomatology, which was strongly associated with report of general anxiety. Parents' and children's reports of posttraumatic symptomatology were not significantly correlated. At follow-up, PTSD symptomatololgy had decreased, but $44 \%$ of children remained symptomatic. Given that PTSD symptomatology was a common consequence of RTA in children, the provision of systematic assessment and, where appropriate, implementation of intervention strategies is indicated. The findings of this study, will be discussed with reference to those with and without mild traumatic brain injury.

\section{Psychosocial Adjustment Following TBI in Children: A Cognitive-Behavioural Formulation}

Tim Hannan, R. Brunsdon and Kath Bakker

University of Sydney

\begin{abstract}
cognitive-behavioural formulation of the psychosocial sequelae of trauAmatic brain injury (TBI) in childhood is offered, in which a child's social, emotional and behavioural adjustment to TBI is conceptualised as the outcome of the interaction between the demands placed on the child and the availability of effective coping skills and resources. In this model, cognitive deficits secondary to TBI are understood to influence the child's appraisal of the demands presented by everyday living, the range and type of coping strategies available to the child, and the effectiveness with which these are utilised. The adoption of this framework has practical implications for the rehabilitation of the brain-injured child. In highlighting the range of factors contributing to psychosocial adjustment, the model encourages a comprehensive approach to assessment of problematic behaviours, emotions and social interactions. Further, this cognitive-behavioural framework provides a theoretical basis for the design and implementation of effective interventions.
\end{abstract}


SYMPOSIUM 6: BEYOND ASSESSMENT: ISSUES IN THE MANAGEMENT OF DEMENTIA

\section{Beyond Assessment: Issues in the Management of Dementia}

Anne Unkenstein

Melbourne Extended Care and Rehabilitation Service, PO Box 7000, Carlton South, VIC 3053

Lucy Smith

Melbourne Extended Care and Rehabilitation Service, PO Box 7000, Carlton South, VIC 3053

The Cognitive, Dementia and Memory Service (CDAMS) in Victoria provides comprehensive assessment for people with dementia. Importantly, this service also focuses on issues of dementia management. An integral part of a visit to the CDAMS is a 'feedback' session. The aim of this session is to feed back the results of various investigations that have taken place at previous visits to the service. In many cases the results indicate a diagnosis of dementia, and this is often the first time the person has been diagnosed. It is often difficult to know when and how to hand over this information to patients and their carers. This session outlines various approaches to feedback, drawing on actual clinical situations to illustrate the complexity of this issue.

The cognitive and behavioural disturbances that may accompany a dementing illness often raise questions concerning an individual's capacity to make independent decisions regarding financial and legal matters, lifestyle choices, accommodation or care needs. Assessment through the CDAMS is often sought to assist in determining an individual's capacity to make dependent decisions, and to formulate appropriate management plans as a result. A number of issues associated with the assessment of decisionmaking capacity and the legal framework for appointment of 'substitute decision-makers' are discussed, using case material to illustrate approaches to assessment and common dilemmas faced by clinicians.

\section{The Role of the Neuropsychologist in the Cognitive Dementia and Memory Service (CDAMS), Kingston Centre}

Louise Dillon, R.A. Howell and B. Workman

Kingston Centre, Warrigal Road, Cheltenham, VIC 3192

The assessment process of the CDAMS, Kingston Centre (established July 1998) typically includes home visit by a member of allied health, and clinic visits for interview with a geriatrician, psychiatrist, and neuropsychologist. Each case is then reviewed at a team meeting, and diagnosis and management plans are formulated; the format for family feedback which follows is also established.

To date, our CDAMS team has found neuropsychology opinion to be integral to the process in most cases. Nevertheless, current funding arrangements preclude neuropsychological involvement in all appropriate cases in most CDAMS. Our aim was to document the unique contribution of the neuropsychologist to the CDAMS in order to provide empirical data for support funding. 
Patients: 50 patients who had completed the CDAMS process, including family feedback in a serial order were selected retrospectively.

Procedure: In addition to demographic data and final diagnoses, 11 variables known to impact on diagnosis and management plans were selected for evaluation. The final reports of CDAMS team members involved in each case were examined, and remarks on any of the selected variables were noted. In addition, each team member's conclusion and recommendations were noted.

Results: The available data will be presented. For reasons which will be discussed, it was decided that the above methodology was flawed, and would not facilitate a valid explication of the original aim.

The role of each team member is currently well defined within our service, ultimately impacting on reporting styles for each professional. Thus, when brief written reports are the data source, it is inaccurate to surmise that variables not noted have not been considered. Moreover, the final diagnosis and management plans are ultimately formulated at the team meeting, and the specific contribution of each professional to decisions is not specified. Nevertheless, the team continues to report that neuropsychological involvement is important in most cases and the need to provide supportive quantitiative evidence to support this remains. Two such cases will be presented briefly.

We invite discussion from the audience about the best way to document the important role of the neuropsychologist in CDAMS.

\section{Changing the Philosophy of Dementia Assessment. A New Service for the Assessment and Management of Problems Related to Dementia}

Michelle Mew, Debbie Senior and Gloria Smith-Tappe

Sunshine Hospital, 176 Furlong Road, St Albans, VIC 3021

$\mathrm{T}$ The Cognitive, Dementia and Memory Service (C.D.A.M.S.) is a specialist service set up throughout Melbourne and regional Victoria. C.D.A.M.S. provides a multidisciplinary assessment, education, and advisory service for individuals in the early stage of dementia. Clients attending this service receive comprehensive medical, neuropsychological, speechlanguage, and psychosocial assessment, feedback and education. Following the assessments, the occupational therapist and speech pathologist can visit the client at home to offer practical suggestions and advice. Counseling and support may also be necessary to assist the client and their family with issues identified during the initial assessment.

C.D.A.M.S. are generally co-located with other aged care service providers to maximise their use of existing infrastructure and administrative support. The establishment of strong links with G.P.'s, local councils, and the Alzheimer's Association, has been necessary, as an important role of C.D.A.M.S. is to refer clients on to other aged care service providers.

This session will highlight the issues associated with setting up a new service, particularly in the Western suburbs in Melbourne, which has a large population of residents from non-English speaking backgrounds. Issues which frequently arise include financial planning, driver assessment, and coping with the cognitive, personality, and behavioural changes associated with dementia. The use of new medications for the management of symptoms associated with Alzheimer's disease also reinforces the importance of early assessment and diagnosis. Early intervention provides opportunities to 
maximise the client's strengths and abilities in light of their cognitive deficits. Practical cognitive interventions offered by occupational therapists and speech therapists to assist the client and their carers will be demonstrated through case examples.

\section{POSTERS}

\section{The Rey-Osterrieth Complex Figure Test: An Organisational Strategy Score for Pediatric Populations}

Peter Anderson, Vicki Anderson and Jenny Bradshaw

Department of Psychology, Royal Children's Hospital, Flemington Road, Parkville, VIC 3052

Jumerous methods for evaluating organisational strategy on the ReyOsterrieth Figure (RCF) have been developed, although no system has gained widespread acceptance. The Rey Complex Figure Organisational Strategy Score (RCF-OSS) was devised for pediatric populations to cater for developmental variability. A sample consisting of 376 children aged between 7.0 and 13.11 years was recruited from schools for the standardisation of RCF-OSS. The RCF copies were scored retrospectively by 4 independent raters according to the RCF-OSS instructions and the level of agreement varied between .88 and .93 . As expected, RCF-OSS correlated moderately with copy and recall accuracy. The RCF-OSS appeared to be assessing a distinct aspect of performance and its relationship with traditional EF measures was curvilinear. Considerable age-related variation in organisational strategy was observed. Correspondence: Peter Anderson, Department of Psychology, Royal Children's Hospital, Flemington Road, Parkville, Victoria, 3052, Australia.

\section{Age Deterioration, Brain Injury and the Recognition of Facial Expression}

Geoffrey A. Fox

Clinical Neuropsychologist, 38 Watonga Street, Pt Macquarie, NSW 2444

Cudies show that most observers may correctly identify the emotion being experienced by a person by observing that person's facial expression. Young has argued that studies have uncovered forms of "emotion recognition impairment", with recognition of fear and anger especially affected by amygdala damage, and disgust also affected by brain injury. Using Young's 30 computer-manipulated images, this research analysed the performance of 143 subjects. Of these, 60 were controls, 49 were victims of mild traumatic brain injury, and 34 were intellectually handicapped or victims of dementia. The age range was from 15 to 83 years. Each person was asked to identify which of 6 feelings (Happy, Surprised, Afraid, Sad, Disgusted, and Angry) was being expressed in each of the 30 images (presented in random order). There were no significant differences due to sex. Age contributed to significant differences in correctly identifying Afraid, Sad, and Disgusted. Analysing the results of the three groups, there were significant differences on Happy, Sad, Disgusted and Angry. Further analyses using an overall accuracy score evaluated the effects of increasing age, and of group membership. The Total Score was a significant factor in evaluating the effects of age and pathology. It is concluded that this short test is sensitive to the effects of both normal and pathological aging. The results appear to add to recent research by Young and colleagues that brain disor- 
der involving the limbic-prefrontal axis affects the perception of emotion communicated through facial expressions. An interesting case study of a woman who suffered a significant injury to the coccyx is presented. The results suggest that a serious injury suffered to the coccyx in a fall may have contributed to a brain disorder.

\section{Memory for Song in Amnesia: Preliminary Findings}

\section{Catherine Haslam}

The Canberra Hospital and the Australian National University, c/o Psychology Department, the Canberra Hospital, PO Box 11, Woden, ACT 2606

$\mathrm{D}$ espite the profound nature of the memory impairment in amnesia, it has been suggested that these patients are capable of remembering songs. Some references have been made to this ability in the literature, yet there has been no systematic investigation of this phenomenon in amnesic patients. In this paper, results from a pilot study investigating memory for song in an amnesic patient will be presented. This patient was capable of accurately distinguishing between old and new lyrics and melodies as well as combination of these components in explicit testing conditions. Furthermore, she was able to provide (i.e., sing) the next line of a melody when presented with random excerpts from studied songs. However, ability to generate and recognise the next line of lyrics was no better than chance. Based on these preliminary findings it appears that in amnesia music can play an important role in supporting familiarity with melody as well as lyrics. Results also suggest that while specific or detailed memory for lyrics was dependent on melody, the opposite was not true.

\section{Neuropsychological Profile of Three Children with Subcortical Band Heterotopia}

Rani Jacobs, Vicki Anderson and A. Simon Harvey

Department of Psychology, Royal Children's Hospital, Flemington Road, Parkville, VIC 3052

Subcortical band heterotopia $(\mathrm{SBH})$ is a neuronal migration disorder characterised by a continuous band of heterotopic grey matter between the cortex and ventricular walls and separated from it by a thin layer of white matter. Current research has a neurological focus with little discussion of cognitive abilities. Three children diagnosed with SBH based on MRI scans are described in this presentation. Reports of developmental milestones were within normal limits, detailed neuropsychological assessment revealed significant impairments with the most prominent feature being slowed speed of processing. Children performed below age expectations on shifting and divided attention and executive function tasks. One child also employed poorer problem solving strategies than expected for her age. Despite appearing functionally intact in early childhood, these processing deficits are likely to impact on later skill development.

\section{Relational Processing and Executive Function Development}

Toni J. Jones

The University of Queensland, School of Psychology, St Lucia, Brisbane, QLD 4072

Two issues frequently discussed in the executive functioning literature will be considered in this paper. The first subject is the well-noted need to identify and describe specific cognitive processes underlying executive 
functioning. It will be suggested that for the source or sources of change in performance to be identified, specific cognitive processes associated with executive functioning need to be isolated. The second related topic concerns the relationship between mental representations, relational processing, and executive functioning. Many renowned researchers have identified the importance of mental representations or models in executive functioning, while other leading researchers including Luria (1973), and Horn and Cattell (1966), have suggested that relational processing also plays a key role in executive functions. It will be suggested that increasing capacity to process complex relational information influences the ability to generate accurate mental models, which in turn influences the efficiency of cognitive processes (such as response inhibition) which underlie executive functioning. Halford, Wilson and Phillips (1998) have developed a metric which identifies the structural complexity of a task or situation in terms of the number of elements and relations that need to be jointly processed in order to generate accurate mental representations. Robin and Holyoak (1995) have reviewed the essential features of executive or pre-frontal functions such as problem-solving, abstraction, attentional control and response inhibition using Halford et al's metric. Waltz and his colleagues (1999) used Halford et al's metric in a neuroimaging study which identified differential relational reasoning deficits in patients with prefrontal deficits in comparison to patients with anterior temporal lobe damage. Findings from both of these studies support the essential role of relational processing in executive functioning. The implications of these findings in terms of current measurement of executive functioning and future research will be discussed.

\section{The Nature of Social Support Needs for People with Traumatic Brain Injury Across Different Life Stages - A Qualitative Examination}

Melissa Kendall and Elizabeth Kendall

Centre for Human Services, Griffith University, Logan Campus, Nathan, QLD 4111

$\mathrm{W}$ hile the literature examining social support following traumatic brain injury (TBI) is considerable, there is a noticeable lack of qualitative studies examining the nature of social support needs for people of different ages. The aim of the current study was to examine the social support needs of people of different ages following TBI through a qualitative approach. A total of 20 people with TBI participated in semi-structured interviews. Participants were presented a range of different situations and asked to describe the ideal type of support they would like to receive in that situation and the people that they would ideally like to provide that support. Participants were also asked to describe the actual type of support they had received in that situation and who had provided it.

Preliminary findings have shown that people of all ages have a need for emotional, practical and informational support from a variety of people. While all types and sources were seen as important, there were differences found across people of different ages. While younger people with TBI tended to show a greater need for emotional support such as someone to provide reassurance and worth, older individuals had a greater need for practical support such as assistance with daily activities and informational support such as advice and guidance. Furthermore, where younger people required more support from peers, older individuals placed more importance on family support. The support actually received by participants was described, in most cases, as significantly less than what was needed. 
These findings have implications for the development of social support problems for people with TBI. Rehabilitation professionals need to consider the particular support networks available to people with TBI and focus on identifying age-appropriate avenues for the development of social support.

\title{
The Clinical Sensitivity of Brief Evaluation of Cognitive Functioning: A Multidisciplinary Approach
}

\author{
Natasha Lannin and Mary Hennessy \\ Townsville General Hospital, Occupational Therapy Department, PO Box 670, Townsville, QLD 4817
}

\begin{abstract}
$\mathrm{T}$ he assessment of cognitive impairment following acquired brain injury (ABI) is a complex and critical issue due to the potential long term impact on an individual's personal, social and occupational abilities. Clarification of the presence and severity of differential cognitive impairment is dependent on accurate assessment procedures. Current high referral levels in general medical and specialist settings reflect this need, with the demand for cognitive assessments presenting a time management challenge for allied health professionals. The issue of cost and time effective, efficient and clinically sensitive assessment procedures is therefore paramount. Currently, there is limited information available relating to the reliability, validity and sensitivity of cognitive screening assessments used by clinical psychologists, neuropsychologists and occupational therapists in the acute medical setting. This research examined the efficacy of neuropsychological and occupational therapy cognitive screens in the early detection and costeffective management of individuals following brain impairment. This study examined performance on five brief evaluations of cognitive function, including the Cognitive Assessment of Minnesota (Rustad, DeGroot, Jungkunz, Freeberg, Borowick, \& Wanttie, 1993), Barry Rehabilitation Inpatient Screening of Cognition (Barry, 1989), Standardised Mini Mental Status Examination (COGNISTAT) (San Fransisco Neuropsychiatric Associates, 1987), and the Brief Neuropsychological Cognitive Examination (BCNE) (Tonkonogy, 1997). Preliminary results and data will be presented and the implications of these findings for research methodology and clinical practice will be discussed.
\end{abstract}

\section{Clock Drawing as a Screening Tool for Alzheimers Disease}

Cressida McCuspie-Moore, Michael Saling and Vicki Anderson

18 Russell Street, Northcote

Tn geriatric psychology and psychiatry, the Clock Drawing Test (CDT) has long been used as a cognitive screening instrument, particularly for dementia of the Alzheimer's type (DAT). In spite of this, research addressing which particular aspects are responsible for its sensitivity has been limited. The general aim of the current study was, therefore, to investigate the reasons subjects with DAT fail on the Clock Drawing Test (CDT) by comparing their performance to a group of cognitively intact age- and education-matched normal control (NC) subjects.

Fifteen subjects with a diagnosis of mild to moderate DAT (MMSE range 18-28) were recruited from an outpatient Impaired Cognition Clinic. In addition, 15 normal control subjects were recruited via advertisements within the hospital or by approaches to spouses of DAT subjects. These two subjects groups were assessed using the CDT, a range of clock related tasks 
(including time setting and time reading), as well as a number of standard neuropsychological measures of general intellectual functioning.

On the standard CDT, DAT subjects were generally impaired compared to NC subjects and also demonstrated a different pattern of errors, reflecting a breakdown in the semantic knowledge about clocks and time representation. Subjects with a diagnosis of DAT performed significantly worse than normal control subjects on many, but not all, of the clock related tests. DAT subjects were as able as NC subjects to copy a line drawing of a clock and to tell the time in a forced choice format. On all other clock related tasks, including measures of time telling and setting, DAT subjects were significantly impaired. Factor analysis of performance on the clock tasks revealed a two-factor solution suggesting that successful performance of these tasks is dependent on both semantic knowledge of what constitutes a clock (ie the features of a clock) and of how time is represented, as well as visuoconstructional ability.

In summary, the CDT was in the current study verified as a clinically useful instrument to distinguish between early DAT and normal cognition at the group level. Performance on the CDT and other clock related tasks is heavily dependent on semantic memory, even early in the course of DAT.

\section{Children with ADHD: A Study Of Self-Management and Family Support Programme}

Amy Scholes and Alan Tucker

Victoria University, Department of Psychology, St Albans Campus, PO Box 14428, MCMC 8001

ttention-Deficit/Hyperactivity Disorder (ADHD) is a commonly occur-
ring developmental disorder yet it continues to be a disorder which
stimulates much debate regarding its appropriate treatment and prognosis.
Stimulant medication continues to be the treatment of choice, but several
recent studies suggest that most effective outcomes would be elicited by the
addition of an appropriate behaviour intervention. In particular, it has been
noted that interventions that combine "behavioural" techniques (eg. rein-
forcement, modeling) with "cognitive" strategies (designed to enhance self-
monitoring, self-evaluation, and self-reinforcement) directed as actual
problems rather than analogue materials could be of most benefit. To further
facilitate management strategies family support is also recommended. This
paper reports an investigation of the utility of self-monitoring intervention
implemented in conjunction with family counseling sessions. Four families
with a child with ADHD between the ages of 6 and 11 years were investi-
gated using a multiple single case design. Three problem behaviours were
identified by the families to be targeted for the intervention. A self-moni-
toring technique was taught to the children using modeling and reinforce-
ment. The child's self-monitoring and behavioral performance was recorded
by parents on a daily basis for a baseline period of one week and for a six
week intervention period. Children were also observed engaging in relevant
behaviours in the clinic. Other measures include the Rowe Behaviour
Rating Inventory, the Family Environment Scale, and a parent evaluation
form. A two month post intervention follow-up was conducted for each
family, followed by an 8 month post intervention follow-up. Preliminary
data analysis suggest that children showed improved self-monitoring and
behaviour for the identified problem areas. Parent evaluations of the inter-
vention were consistently positive and the family environment was found to
have changed its pattern and emphases across the time period. Improved 
behaviour, positive evaluation and family environment change remained

evident two months subsequent to completion of the intervention.

\title{
Spatial Process in the Visual and Tactual Modalities: The Spatial Coordinates System
}

\author{
Ian Stuart
}

Royal Talbot Rehabilitation Centre, (Campus of ARMC), Yarra BIvd, Kew, VIC 3101

Tt is well known that the visual and tactual modalities are specialised for the recognition of objects, and disorders of this process are identified as the agnosias. Semmes (1965) suggested that the representation of more complex spatial information is not tied to a particular modality, but occurs in a supramodal fashion. The aim of the study was to test this hypothesis.

A new test of tactual learning, the Tactual Learning Test (TLT), was developed and, with the Visual Reproductions of the WMS-R (VisRep), was given to a group of one hundred and nine subjects of mixed aetiology. This included seven subjects who had suffered damage to the right parieto-occipital area and whose copy of the Rey complex figure showed a fragmented appearance. There were seventeen control subjects.

A single-factor between subjects ANOVA model was tested, with five clinical sub-groups comprising the single nominal predictor. This showed a pattern of results across the five sub-groups for both dependent variables and a notably poor performance on both tests for the subjects in the Fragmented sub-group. This supports the hypothesis that spatial abilities are not represented in a modality-specific manner.

A previous study, Stuart (1996), showed that children who were blind from birth had the capacity to succeed on the Tactual Learning Test. It is concluded, therefore that the visual modality is not essential for the development of complex spatial abilities. It is also concluded that the Spatial Coordinate System is not truly supramodal in nature. The representation of movement of the hands and arms around the body-centred vertical axis forms the basis of the Spatial Coordinate System, with visual information playing a minor role in spatial thought.

\section{Key Findings from a Meta-Analytical Study on Intellectual Functions in Duchenne Muscular Dystrophy}

\author{
Sue Cotton, Nicholas Voudouris and Jacinta Douglas \\ Faculty of Health Sciences, LaTrobe University, Plenty Road, Bundoora, VIC 3083
}

\begin{abstract}
A meta-analytical investigation was conducted reviewing four decades of literature on intellectual functioning in DMD, with the essential findings to be reported here. Individual data from both the Wechsler Intelligence Scales (WIS) and the Standford-Binet, were available for 1146 children with DMD, from 32 studies. The incidence of children with an intelligence quotient (IQ) less than 70 was $34.8 \%$. Full scale IQ, Verbal IQ, and Performance IQ scores were normally distributed with means approximately one-standard deviation below the normal population average of 100 . The mean VIQ-PIQ discrepancy was -5.1. The distribution of scores on the WIS subtests indicated a distinctive profile with weaknesses being Arithmetic, Vocabulary, Comprehension, and Coding. Strengths were notably on the performance subtests. The importance of age and severity of
\end{abstract}


disease will be discussed with reference to intellectual functioning in this population. Recommendations will be also made for further research.

\title{
Polite Lies and Dripping Sarcasm. An Investigation of the Effects of Traumatic Brain Injury on Understanding Natural Conversational Meaning
}

\author{
Skye McDonald and Sharon Flanagan
}

$I_{t}^{t}$ has been established that a significant proportion of adults with severe traumatic brain injury (TBI) have difficulty understanding conversational inferences. However, the vast majority of research has used written samples of conversational exchanges. It is unknown whether such patients have difficulty with realistic conversational exchanges that involve multiple channels of information (voice, facial expression etc.). Nor is it known whether such patients are able to use additional contextual information to their advantage when interpreting conversational inference (e.g. knowing the true feelings of the speaker beforehand),

13 TBI patients and 10 non-brain-injured control subjects viewed 21 vignettes of conversational exchanges in which the primary speaker was (a) sincere (b) lying in order to be polite or (c) being sarcastic. In (b) and (c) additional information was provided to "set the scene" e.g. a visual cue (such as being shown a shot of a book that is covered in scribbles) followed by the comment " $N o$ Rosie hasn't scribbled on my book". Alternatively, there was additional dialogue before or after the main interaction, e.g. two co-workers confiding to each other that a party was truly dreadful, followed by a scene with the party's host in which they claim the party was a great success. Ability to interpret each vignette correctly was assessed via a set of questions.

Despite good performance when asked to judge sincere exchanges. TBI patients were significantly impaired relative to controls in their ability to understand both lies and sarcasm. For sarcasm they failed to understand what the main speaker was feeling, thinking, meaning by what they said, or intending toward the other interlocuter. For lies they had particular difficulty understanding what the speaker was intending and thinking. These results validate previous research and highlight the prevalence of communication disturbances after TBI.

\section{Working Memory in Children with Tourette Syndrome (TS): Dual Task Performance and Sentence Comprehension}

Annemaree Carroll, Veronica Comerford, Maggie Bailey and Gina Geffen

Graduate School of Education, Cognitive Psychophysiology

Laboratory, University of Queensland and Queensland Health

\begin{abstract}
A $\mathrm{n}$ influential model of working memory (Baddeley, 1994) was used as A a conceptual framework for testing the neurological dopamine hypothesis of Tourette Syndrome (TS) that predicts dysfunctions of the prefrontal cortex. We administered a variety of screening measures as well as tests of dual task performance to children with TS ( $N=40,6$ females) and age, sex and IQ matched controls. Parent ratings of TS children (Child Behaviour Check List) indicated many more problems and particularly with the Attention scale, than equivalent ratings by control group parents. Standardised tests purported to measure frontal lobe functioning (Wisconsin Card Sort Test, Tower of London), and specific tests of dual task perfor-
\end{abstract}


mance (TEA-Ch, Visual Tracking and Memory Span Test), were of primary interest. The results indicated no group differences on the Wisconsin Card Sort or Tower of London tests. In the dual task measures, however, children with TS counted fewer spaceship blasts (in either condition of the TEA-Ch) and had reduced digit span in dual task conditions. Dual task decrement was found to be greater when the two tasks were conducted in the same modality, than when they were conducted in different modalities. For verbal memory, TS children showed poorer immediate recall of five common words and took longer to name pictures than controls. In terms of the model, impaired functioning of the phonological loop and the central executive control loop are implicated in TS rather than a generalised deficit of the central executive. Since attention deficit hyperactivity disorder frequently cooccurs with TS, further work is required to determine whether these impairments are TS specific.

\section{Executive Functioning in Children with Tourette Syndrome}

Maggie Bailey, Annemarree Carroll, Gina Geffen and Veronica Comerford

Cognitive Psychophysiology Laboratory and School of

Education, University of Queensland, Queensland Health

The dopamine hypothesis of the etiology of Tourette Syndrome (TS) predicts a dysfunctional executive system in children with this disorder. In the present study, the executive functions of children with TS $(N=44,6$ females), and age and IQ matched controls with the same sex representation were compared. The results of three commonly used tests, Stroop Colour Word Test, Contingency Naming Test and Trailmaking Test are reported. On the Stroop and Contingency Naming tests, the performance of both groups showed significant linear decline with increasing complexity. Similarly, children in both groups took significantly longer to complete Trails B than Trails A. Children with TS read fewer words than controls in the Stroop timed word reading task, but there were no significant differences between the groups on the inhibition measures nor on the more complex conditions of the tests. These results are consistent with the findings on the same children (Carroll, Geffen, Bailey et al) in which no group differences were found on the Wisconsin Card Sort or Tower of London tests, but TS children showed poorer immediate recall of five common words, counted fewer spaceship blasts (in either condition of the TEACH), had reduced digit span in dual task conditions, and took longer to name pictures than controls. In terms of Baddeley's (1994) model of working memory, impaired functioning of the phonological loop and the central executive control loop are implicated in TS rather than a generalised deficit of the central executive.

\section{Effect Of Normal Ageing On Sleep, Sleepiness And Cognitive Performance}

Kate Crowley

Department of Psychology, University of Melbourne

Changes in the sleep-wake pattern are considered among the hallmarks of biological aging. Many studies have shown that the elderly complain of difficulties in initiating and maintaining sleep as well as elevated levels of daytime sleepiness and reduced cognitive function. A problem with the interpretation of these studies is that they rarely if ever exclude subjects with substantial levels of pathology that tend to correlate with aging, for exam- 
ple cardiovascular or neurological disease. This study investigated the relationship of subjectively defined levels of sleep and daytime sleepiness with performance on a wide range of cognitive measures known to be sensitive to sleep loss. Thirty neurologically healthy elderly individuals (mean age $75.1 \pm 5.85$ ) and 30 young participants (mean age $19.3 \pm 3.34$ ) participated in the study. All elderly participants were carefully screened with reference to rigorous exclusion criteria relating to health conditions identified as potentially effecting the integrity of the Central Nervous System. Data consisted of sleep diaries, the Epworth Sleepiness Scale and the Karolinska Sleepiness Scale. The performance variables included measures of attention, verbal memory and executive function. The major findings were: an absence of differences in self-reported measures of sleep and daytime sleepiness between young and old subjects and an absence of neuropsychological test performance differences between the two groups, with the exception of measures involving psychomotor speed. Results suggest that in neurologically healthy adults, aging is not associated with self reported changes in the sleep process or increased daytime sleepiness.

\title{
Cognitive Functioning in the Neurologically Healthy-Aged: Some Initial Findings
}

Tracey Wardill

Department of Psychology, University of Melbourne

\begin{abstract}
need to better understand the impact of the aging process on cognitive functioning is highlighted throughout the literature on normal aging. One means of improving our understanding of the impact of age on cognition would be to establish reliable performance data for commonly used neuropsychological tests. Such data are important in the clinical setting as they assist Neuropsychologists to accurately diagnose cerebral pathology in the aged. There is currently a lack of performance data for age groups over 65 years, particularly for the Australian population. Previous attempts to obtain such data reflect a lack of rigor in the selection of subjects and small sample sizes. Research in progress at the Austin and Repatriation Medical Centre in Melbourne, is addressing these issues. The project is examining a number of areas of cognitive functioning, one of which is memory. Memory deficits in the age can herald the onset of various pathological conditions, including the most common form of dementia, Alzheimer's disease. An understanding of normal memory functioning in the older population, therefore, is vital. This poster will outline this research project and present data from the first 159 subjects on a test that is being commonly used in the assessment of memory in the aged, the Hopkins Verbal Learning Test.
\end{abstract}

